\title{
Expression of a neural stem/progenitor cell marker nestin in salivary glands
}

\begin{abstract}
Salivary glands have the ability to regenerate, yet the origin and markers of stem/progenitor cells involved in this regeneration are still unclear. We have previously reported that the expression of cytokeratin 5, which is considered as a marker of both basal/myoepithelial and progenitor cells in salivary glands, was enhanced by tissue injury. In this study, to identify more specific markers for progenitor cells of salivary glands, we examined the expression and localization of nestin in damaged tissues by duct ligation. Changes in mouse parotid glands after unilateral obstruction of the main excretory duct were analyzed histologically, with immunoblot analysis, and with immunohistochemical staining. Atrophy of acinar cells was observed on the side of duct ligation with hematoxylin and eosin staining. On the other hand, the contralateral side and sham-operated salivary glands did not change morphologically. The amount of nestin protein on the ligated side was increased and was significantly higher than on the contralateral side at day 4. Immunohistochemical staining demonstrated nestin-positive cells in the ducts and the atrophied acinar cells and around them. And some cells expressed both nestin and cytokeratin 5. Ki-67-positive cells were also increased at day 4 on the ligated side, indicating that proliferation of progenitor cells begins simultaneously with the increase in nestin. These results suggest that nestin may be a useful marker of progenitor cells in salivary glands.
\end{abstract}

Keywords: salivary gland, duct ligation, nestin, progenitor marker
Volume 4 Issue 2 - 2017

\author{
Megumi Yokoyama, Osamu Katsumata-Kato, \\ Hajime Sakurai, Hiroaki Ogawa, Junko Fujita- \\ Yoshigaki \\ Department of Physiology, Nihon University School of Dentistry \\ at Matsudo, Japan
}

\begin{abstract}
Correspondence: Megumi Yokoyama, Department of Physiology, Nihon University School of Dentistry at Matsudo, 2870I, Sakae-cyo-nishi, Matsudo-shi, Chiba, 27I-8587, Japan, Tel +81 473609326 , Fax +81473609327.

Email yokoyama.megumi02@nihon-u.ac.jp
\end{abstract}

Received: August 25, 2017 | Published:September 19, 2017

\section{Introduction}

Salivary glands are important for secretion of saliva, which is essential for bolus formation, swallowing and maintaining a healthy oral cavity. ${ }^{1}$ Salivary glands consist of acinar and duct cells. Injury to salivary glands, such as the damage that occurs following radiation treatment for head and neck cancer, causes a decrease in the number of acinar cells and the ability of the glands to produce saliva. ${ }^{2,3}$ After tissue injury ceases and if the damage is not too severe, the proportional volume of acini increases, and the salivary gland recovers its functions. ${ }^{4,5}$ The salivary gland has the ability to regenerate, but the origin of regenerated salivary gland cells and progenitor cell markers are still incompletely understood. Identification of a marker protein in salivary glands will be important for designing therapeutic strategies to restore function of injured salivary glands.

We have previously reported that the expression level of cytokeratin 5 in parotid glands increased during tissue injury caused by ligation of the main excretory duct. ${ }^{6}$ Because cytokeratin 5 has been reported as a marker of progenitor cells in salivary glands, ${ }^{7}$ increase of cytokeratin 5 in response to tissue injury can be interpreted as an activation and proliferation of stem/progenitor cells to replace damaged cells. However, cytokeratin 5 is also expressed in basal cells of ducts and myoepithelial cells, and identification of more specific markers for progenitor cells is desirable.

Nestin was first described as a neuronal stem/progenitor cell marker $^{8}$ and is also a useful progenitor marker in pancreatic exocrine cells. ${ }^{9}, 10$ Because the tissue structures and functions of the exocrine pancreas and salivary gland show some similarities and stem cell populations from salivary glands and the pancreas have similar phenotypes and characteristics, ${ }^{11}$ we hypothesized that nestin is also a useful progenitor cell marker of the salivary gland. Therefore, in this study, we induced tissue injury in salivary glands by obstruction of the main excretory duct in order to promote activation and increase of stem/progenitor cells and then examined nestin expression.

\section{Materials and methods}

\section{Experimental animals}

Male C57BL/6 mice at 8 weeks of age were used throughout the present experiments. They were obtained from Sankyo Labo Service Corporation, Inc. (Tokyo, Japan) and maintained in our animal facility in standard conditions (12hour light, 12hour dark schedule) with free access to food and water. Under inhalation anesthesia with $3 \%$ sevoflurane, an incision was made on the skin on the left side of the cheek. Surrounding connective tissues were separated from the main excretory duct under a microscope, and the duct was ligated with a microclip (Mizuno Ikakogyo Co., Ltd., Tokyo, Japan). The incision was closed with polyamide sutures. At 4, 7, and 10days after ligation, the animals were re-anesthetized, and the parotid gland was excised. The experiment conformed to the institutional guidelines for the use of experimental animals and was approved by the Experimental Animal Ethical Committee of Nihon University School of Dentistry at Matsudo (AP11MD003).

\section{Hematoxylin and eosin staining}

The parotid gland was fixed in $10 \%$ formalin in phosphatebuffered saline, embedded in paraffin, cut into $4-\mu \mathrm{m}$-thick sections, and mounted on slides. For observation of general morphology, tissue sections were stained with hematoxylin and eosin. All histological sections were examined by light microscopy using an Olympus BX51, and images were acquired with the Olympus DP12 system. 


\section{Immunoblot analysis}

Parotid glands were homogenized in lysis buffer (20 mM HEPES, $\mathrm{pH} 7.4$ containing $0.1 \%$ Triton X-100, $1 \times$ Complete protease inhibitor cocktail). For immunoblot analysis, $10 \mu \mathrm{g}$ protein from samples of ligated and non-ligated glands and $20 \mu \mathrm{g}$ protein from sham-operated samples were separated with $7.5 \%$ SDS-PAGE and transferred to a membrane (HybondTM-LFP; GE Healthcare, Buckinghamshire, UK). After blocking with Blocking Agent (GE Healthcare) at room temperature for 1hour, the membranes were incubated with anti-nestin antibody (ab6142, Abcam, Tokyo, Japan, 1:500). Immunoreactivity was determined with an ECL Plex Western blotting kit (GE Healthcare). Images were acquired with Typhoon Trio (GE Healthcare), and fluorescence intensities were quantified with Image Quant TL software (GE Healthcare).

\section{Immunohistochemical staining}

Nestin staining was performed using the Histofine Mouse Stain kit (Nichirei Biosciences Inc., Tokyo, Japan). Antigen retrieval for nestin and cytokeratin 5 staining were performed by boiling sections for 20min, and Ki-67 staining was treated for 35min in Dako REALTM Target retrieval solution (Dako Denmark A/S, Glostrup, Denmark).Endogenous peroxidase activity was blocked by treating the sections with $30 \%$ hydrogen peroxide in methanol for $10 \mathrm{~min}$. The sections were incubated with anti-nestin monoclonal antibody (Abcam, 1:100) or anti-cytokeratin 5 polyclonal antibody (BioLegend lnc, Massachusetts, USA, 1:200) or anti-Ki-67 polyclonal antibody (Abcam, 1:100) for 1 hour at room temperature. For cytokeratin 5 and Ki-67 staining, the ChemMate Envision secondary antibody (Dako Cytomation, Glostrup, Denmark) was used. The secondary antibody for nestin was that included in the Histofine Mouse Stain kit. Staining was visualized with 3, 3'-diaminobenzidine (DAB; MERCK KGaA, Darmstadt, Germany). Subsequently, the sections were counterstained with Mayer's hematoxylin and mounted. The percentage of Ki-67 positive cells were counted in 3 fields (magnification $\mathrm{x} 400$ ) for each sample.

\section{Statistical analysis}

All values are reported as the mean $\pm \mathrm{SD}$. Data were analyzed using Student's t-test. $\mathrm{P}<0.05$ was considered significant.

\section{Results}

\section{Histological changes}

To confirm the effect of duct ligation, we observed histological changes in the ligated and non-ligated sides of parotid glands. On the ligated side, gradual atrophy of acinar cells was observed (Figure 1A-1C). Connective tissue and inflammatory cell infiltration were increased on the ligated side over time (Figure 1B) (Figure 1C). The non-ligated side of parotid glands showed normal morphology at all time points (Figure 1D). The morphology of sham-operated glands was the same as that on the non-ligated side at each time point (data not shown).

\section{Expression level of nestin}

Removal of the ligation of the duct induces regeneration of parotid glands in 7 days, ${ }^{5}$ suggesting that progenitor cells are activated and proliferate during ligation. Progenitor cell markers may decrease after recovery of salivary glands. Therefore, we examined the change in expression levels of nestin during duct ligation. The amount of nestin protein on the ligated and non-ligated sides of glands was compared with that in sham-operated glands. Nestin demonstrated an upward trend on the ligated side at all time points compared with the non-ligated side and sham-operated glands. Nestin was slightly increased, even in the non-ligated gland compared with that of shamoperated glands, but the amount was lower than on the ligated side (Figure 2A). Next, we quantified the amount of nestin protein and performed statistical analysis. Nestin expression on the ligated side was significantly increased at day 4 compared with the non-ligated side. On the other hand, no significant differences between the ligated and non-ligated sides were observed at days 7 and 10 (Figure 2B).
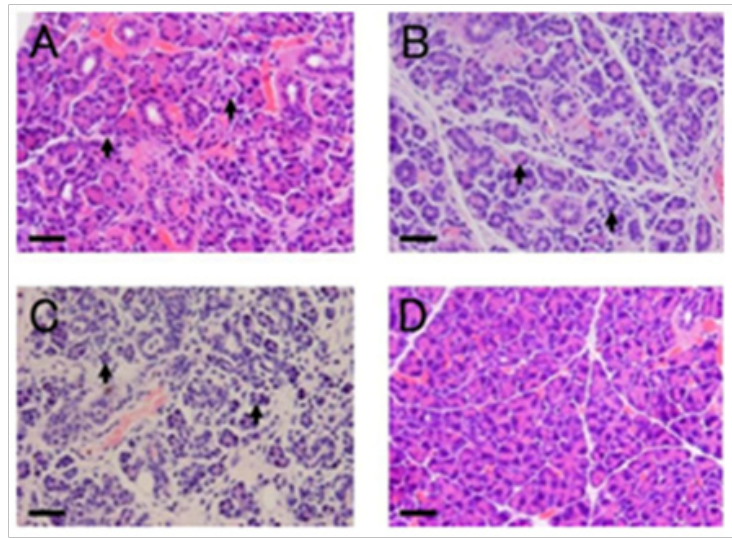

Figure I Morphological changes after ligation of the main excretory duct (hematoxylin and eosin staining).

A-C, Atrophy of acinar cells (arrow) was observed on the duct-ligated side at days $4(\mathrm{~A}), 7(\mathrm{~B})$, and $10(\mathrm{C})$.

D, Representative morphology of the non-ligated side (Day 7).

Scale bars, $30 \mu \mathrm{m}$.

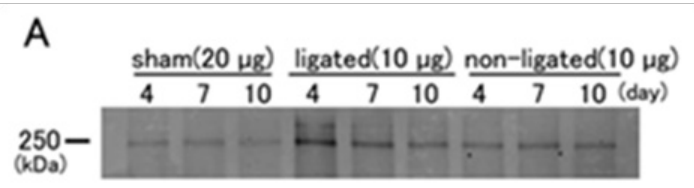

B

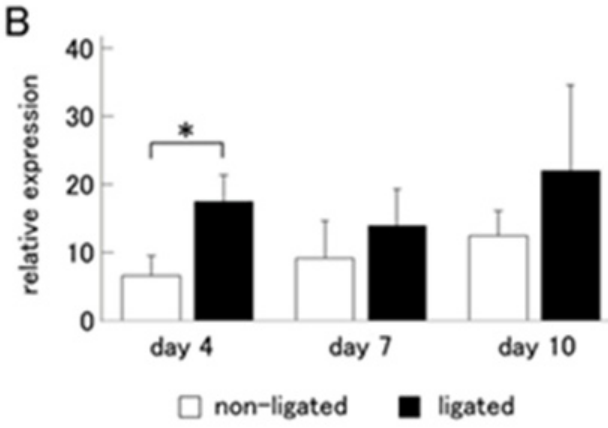

Figure 2 Change in the expression level of nestin in the parotid gland after duct ligation.

A) Because the expression of nestin in sham-operated parotid glands was much lower than in ligated and contralateral sides of glands, 20 $\mu \mathrm{g}$ protein from sham-operated glands was used, whereas $10 \mu \mathrm{g}$ protein from ligated and non-ligated sides was used for western blotting analysis.

B) Nestin expression levels in the ligated and non-ligated parotid glands are shown as values that are relative to that of sham-operated glands. Expression of nestin on the ligated side was significantly increased at day 4 compared with the non-ligated side. The data represent the mean \pm SD $(n=5) . * P<0.05$ compared to the non-ligated side. 


\section{Localization of nestin in the ligated glands}

To identify nestin-expressing cells in injured salivary glands, we performed immunohistochemical staining. On the ligated side of parotid glands, the expression of nestin was observed at all time points. Although the localization was unclear because of cellular atrophy, nestin-positive cells were observed in the ducts, atrophied acinar cells, and interstitial tissue. Some nestin-positive cells seem to be located basally in the gland epithelium (Figure 3A-3C). On the non-ligated side and sham-operated gland, the expression of nestin was not detected at any time points (Figure 1E) (Figure 3D).
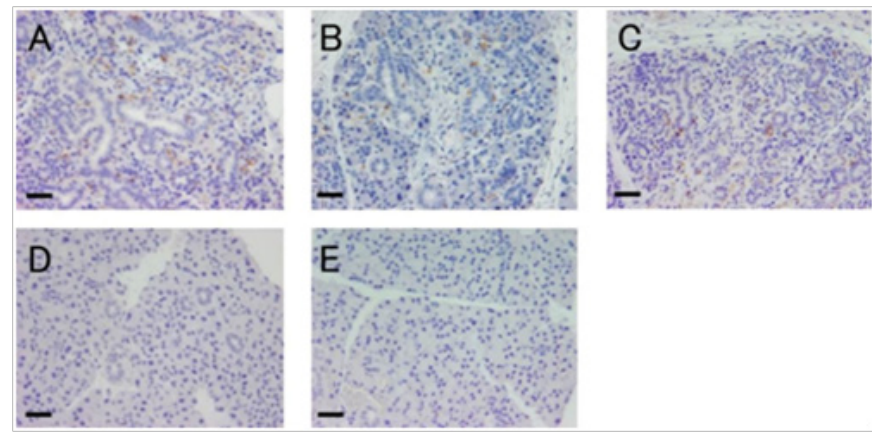

Figure 3 Localization of nestin in the ligated glands.

A-C, Ligated side of parotid glands at days 4(A), 7(B), and $10(C)$.

Nestin-positive cells (brown) were observed in the ligated glands.

$D$, Representative morphology of the non-ligated gland (day 4).

E, Representative morphology of the sham-operated gland (day 4).

Scale bars, $30 \mu \mathrm{m}$.

\section{Expression of cytokeratin $\mathbf{5}$ and nestin in serial sections}

In order to examine whether nestin-positive cells also expressed cytokeratin 5, we performed immunofluorescence double staining by using anti-nestin and anti-cytokeratin 5 antibodies. In that experiment, nestin strongly stained the extracellular matrix and its original signal could not be identified. Therefore, we prepared serial sections of ligated glands and carried out immunohistochemical staining with each antibody. Some cells expressed both nestin and cytokeratin 5 in the same area. On the other hand, some cells were only nestin-positive or cytokeratin 5-positive (Figure 4A \& Figure 4B)
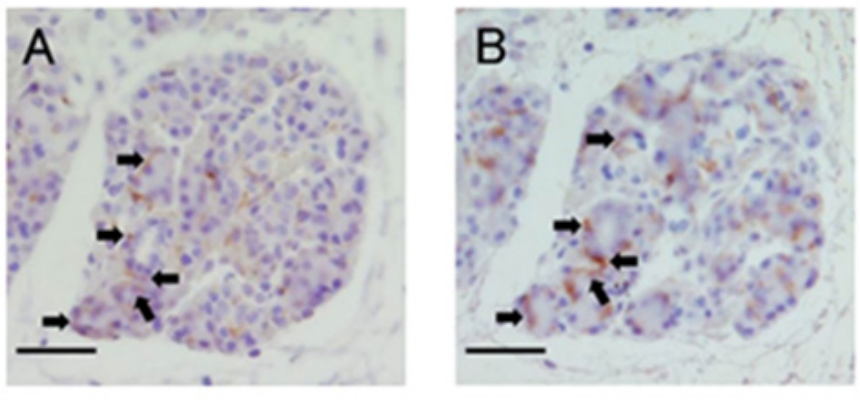

Figure 4 Localization of nestin and keratin 5 in the serial section.

A) Immunohistochemistry staining of nestin in the ligated parotid glands at day 4 .

B) Same area as seen in A, examined for expression of keratin 5 .

Arrows indicate cells that matched in the expression.

Scale bars, $30 \mu \mathrm{m}$.

\section{Expression of the cell proliferation marker Ki-67 in the ligated glands}

As shown in Figure 3, the expression level of nestin was significantly increased at day 4 . Therefore, we focused on day 4 and performed immunohistochemistry for Ki-67, a marker of cell proliferation. Ki-67 was detected in atrophied acinar, duct cells and interstitial tissue (Figure 5A). On the non-ligated side, Ki-67-positive cells were detected in acinar and duct cells (Figure 5B). On the ligated side, Ki-67-positive cells were significantly increased compared with the non-ligated side (Figure 5C).

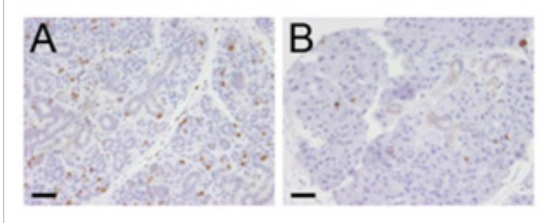

C

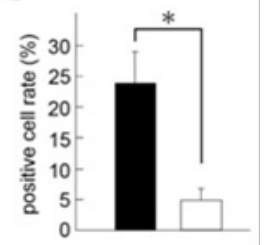

Figure 5 Expression of $\mathrm{Ki}-67$ in the ligated glands at day 4 .

A) Ligated side of parotid glands.

B) Non-ligated side of parotid glands. Ki-67-positive cells (brown) were increased on the ligated side compared with the non-ligated side. Scale bars, $30 \mu \mathrm{m}$.

C) Quantification of Ki-67-positive cells. Positive cell rate was significantly increased in ligated side (black bar) compared to non-ligated side (white bar). The data represent the mean $\pm S D(n=3)$. *P $<0.05$ compared to the non-ligated side.

\section{Discussion}

Duct obstruction of salivary glands in rodents is a commonly used model for studying salivary gland damage. ${ }^{12}$ Salivary gland atrophy is a common consequence of disease and injury, such as radiation treatment, Sjögren's syndrome, and sialadenitis, and a reduction in the number of normal acinar cells causes a decrease in saliva secretion. ${ }^{13}$ During atrophy of salivary glands caused by excretory duct ligation, acinar cells disappear due to apoptosis ${ }^{14,15}$ or dedifferentiation. ${ }^{16}$ In this study, atrophy of acinar cells and inflammatory cell infiltration were observed on the ligated side. The appearance of inflammatory cell infiltration is considered a defense response of the body against obstructed salivary secretion due to ligation of the main excretory duct.

The purpose of this study was to determine progenitor cell markers in salivary glands. We examined whether nestin is expressed in the injured salivary gland following ligation of the main excretory duct. At 4days after ligation, the expression level of nestin was significantly increased on the ligated side. We previously reported that cytokeratin 5 , which is a candidate progenitor cell marker, is increased at days 7 and 10 after injury. ${ }^{6}$ Thus, the expression of progenitor cell markers appears to change during tissue injury and subsequent dedifferentiation of the gland cells. In this study with Ki-67 staining, we verified that cell proliferation had already begun during duct ligation. Presumably, cell proliferation of salivary gland cells is promoted by tissue injury. If the decrease in acinar cells caused by apoptosis and atrophy exceeds proliferation and differentiation, the number of acinar cells in the glands will be reduced and their function to generate saliva will decline.

The amount of nestin protein in the non-ligated glands was increased compared with sham operation, although the change was 
smaller than in ligated glands. Such a response indicates that the nonligated glands were influenced by signals from the opposite injured gland. Identification of such signals may be useful for activation of quiescent progenitor cells. Expression of nestin was detected in the non-ligated side in immunoblot analysis (Figure 2A). But the cell that markedly expressed nestin was not observed in immunohistochemical experiment (Figure 3D). In the ligated side of parotid gland, some cells expressed either nestin or cytokeratin 5 (Figure 4). We previously reported that cytokeratin 5 increased at days 7 and 10 after ligation, which indicates that increase in cytokeratin 5 occurred after nestin expression. The expression pattern of precursor cell markers appears to change in the course of injury. Therefore, we consider that some cells only express one protein.

In vitro, tissue damage induces dedifferentiation of rat parotid acinar cells that is mediated by Src kinase and p38 MAP kinase. ${ }^{17}$ Some progenitor cells are derived from previously differentiated cells via dedifferentiation. ${ }^{18,19}$ In our study, cells other than duct cells, which may be atrophied acinar cells, were nestin positive. Acinar cells in the rodent salivary glands apparently differentiate from intercalated duct cells. ${ }^{20,21}$ Recently, there are reports of the main source of regenerated acinar cells after duct ligation is self-duplication of residual acinar cell, ${ }^{22}$ and normal salivary gland homeostasis results mainly from selfduplication of acinar cells. ${ }^{23}$ Our results suggested that the origin of acinar cells that regenerate after tissue injury may not be limited to duct cells.

\section{Conclusion}

The expression of nestin was induced by tissue injury in salivary glands and some of nestin-positive cells simultaneously expressed cytokeratin 5 . These results suggest that nestin is a progenitor cell marker in salivary glands. Further studies are needed to elucidate whether nestin-positive cells differentiate into acinar cells.

\section{Acknowledgements}

This work was supported by JSPS KAKENHI Grant Number $15 \mathrm{~K} 20564$ and research grants from the Research Institute of Oral Science, Nihon University School of Dentistry at Matsudo and from the Hamaguchi Foundation for the Advancement of Biochemistry.

\section{Conflicts of interest}

The authors have no conflicts of interest relevant to this article.

\section{References}

1. Arany S, Catalán MA, Roztocil E, et al. Ascl3 knockout and cell ablation models reveal complexity of salivary gland maintenance and regeneration. Dev Biol. 2011;353(2):186-193.

2. Vissink A, Kalicharan D, S-Gravenmade EJ, et al. Acute irradiation effects on morphology and function of rat submandibular glands. J Oral Pathol Med. 1991;20(9):449-456.

3. Valdés Olmos RA, Keus RB, Takes RP, et al. Scintigraphic assessment of salivary function and excretion response in radiation-induced injury of the major salivary glands. Cancer. 1994;73(12):2886-2893.

4. Funegård U, Franzén L, Ericson T, et al. Parotid saliva composition during and after irradiation of head and neck cancer. Eur J Cancer B Oral Oncol. 1994;30B(4):230-233.

5. Burford Mason AP, Cummins MM, Brown DH, et al. Immunohistochemical analysis of the proliferative capacity of duct and acinar cells during ligation-induced atrophy and subsequent regeneration of rat parotid gland. J Oral Pathol Med. 1993;22(10):440-446.
6. Yokoyama M, Katsumata-Kato O, Matsuki-Fukushima M, et al. Cytokeratin 5 expression in salivary duct ligation. J Oral Sci. 2012;38(2):96-100.

7. Knox SM, Lombaert IM, Reed X, et al. Parasympathetic innervation maintains epithelial progenitor cells during salivary organogenesis. Science. 2010;329(5999):1645-1647.

8. Lendahl U, Zimmerman LB, McKay RD. CNS stem cells express a new class of intermediate filament protein. Cell. 1990;60(4):585-595.

9. Carrière C, Seeley ES, Goetze T, et al. The nestin progenitor lineage is the compartment of origin for pancreatic intraepithelial neoplasia. Proc Natl Acad Sci USA. 2007;104(11):4437-4442.

10. Esni F, Stoffers DA, Takeuchi T, et al. Origin of exocrine pancreatic cells from nestin-positive precursors in developing mouse pancreas. Mech Dev. 2004;121(1):15-25.

11. Gorjup E, Danner S, Rotter N, et al. Glandular tissue from human pancreas and salivary gland yields similar stem cell populations. Eur $J$ Cell Biol. 2009;88(7):409-421.

12. Woods LT, Camden JM, El-Sayed FG, et al. Increased expression of TGF- $\beta$ signaling components in a mouse model of fibrosis induced by submandibular gland duct ligation. PLoS One. 2015;10(5):e0123641.

13. Silver N, Proctor GB, Arno M, et al. Activation of mTOR coincides with autophagy during ligation-induced atrophy in the rat submandibular gland. Cell Death Dis. 2010;1:e14.

14. Walker NI, Gobé GC. Cell death and cell proliferation during atrophy of the rat parotid gland induced by duct obstruction. J Pathol. 1987;153(4):333-344.

15. Scott J, Liu P, Smith PM. Morphological and functional characteristics of acinar atrophy and recovery in the duct-ligated parotid gland of the rat. J Dent Res. 1999;78(11):1711-1719.

16. Takahashi S, Schoch E, Walker NI. Origin of acinar cell regeneration after atrophy of the rat parotid induced by duct obstruction. Int $J$ Exp Pathol. 1998;79(5):293-301.

17. Fujita Yoshigaki J, Matsuki Fukushima M, Sugiya H. Inhibition of Src and p38 MAP kinases suppresses the change of claudin expression induced on dedifferentiation of primary cultured parotid acinar cells. $\mathrm{Am}$ J Physiol Cell Physiol. 2008;294(3):C774-C785.

18. Velloso CP, Simon A, Brockes JP. Mammalian postmitotic nuclei reenter the cell cycle after serum stimulation in newt/mouse hybrid myotubes. Curr Biol. 2001;11(11):855-858.

19. Pearton DJ, Yang Y, Dhouailly. Transdifferentiation of corneal epithelium into epidermis occurs by means of a multistep process triggered by dermal developmental signals. Proc Natl Acad Sci USA. 2005;102(10):3714-3719.

20. Cotroneo E, Proctor GB, Carpenter GH. Regeneration of acinar cells following ligation of rat submandibular gland retraces the embryonic-perinatal pathway of cytodifferentiation. Differentiation. 2010;79(2):120-130.

21. Schwartz Arad D, Arber L, Arber N, et al. The rat parotid gland--a renewing cell population. J Anat. 1988;161:143-151.

22. Aure MH, Arany S, Ovitt CE. Salivary glands: stem cells, selfduplication, or both? J Dent Res. 2015;94(11):1502-1507.

23. Aure MH, Konieczny SF, Ovitt CE. Salivary gland homeostasis is maintained through acinar cell self-duplication. Dev Cell. 2015;33(2):231-217. 